\title{
A Giant Outburst at Millimeter Wavelengths in the Orion Nebula
}

\author{
Geoffrey C. Bower ${ }^{1}$, Richard L. Plambeck ${ }^{1}$, Alberto Bolatto ${ }^{1}$, Nate McCrady ${ }^{1}$, James R. \\ Graham $^{1}$, Imke de Pater ${ }^{1}$, Michael C. Liu ${ }^{2,3}$, \& Frederick K. Baganoff ${ }^{4}$
}

\begin{abstract}
BIMA observations of the Orion nebula discovered a giant flare from a young star previously undetected at millimeter wavelengths. The star briefly became the brightest compact object in the nebula at $86 \mathrm{GHz}$. Its flux density increased by more than a factor of 5 on a timescale of hours, to a peak of $160 \mathrm{mJy}$. This is one of the most luminous stellar radio flares ever observed. Remarkably, the Chandra X-ray observatory was in the midst of a deep integration of the Orion nebula at the time of the BIMA discovery; the source's X-ray flux increased by a factor of 10 approximately 2 days before the radio detection. Follow-up radio observations with the VLA and BIMA showed that the source decayed on a timescale of days, then flared again several times over the next 70 days, although never as brightly as during the discovery. Circular polarization was detected at 15,22 , and $43 \mathrm{GHz}$, indicating that the emission mechanism was cyclotron. VLBA observations 9 days after the initial flare yield a brightness temperature $T_{b}>5 \times 10^{7} \mathrm{~K}$ at $15 \mathrm{GHz}$. Infrared spectroscopy indicates the source is a $\mathrm{K} 5 \mathrm{~V}$ star with faint $\operatorname{Br} \gamma$ emission, suggesting that it is a weak-line T Tauri object. Zeeman splitting measurements in the infrared spectrum find $B \sim 2.6 \pm 1.0 \mathrm{kG}$. The flare is an extreme example of magnetic activity associated with a young stellar object. These data suggest that short observations obtained with ALMA will uncover hundreds of flaring young stellar objects in the Orion region.
\end{abstract}

Subject headings: open clusters and associations: individual (Orion Nebula Cluster) — stars: flare — stars: formation — stars: magnetic fields

\footnotetext{
${ }^{1}$ Astronomy Department \& Radio Astronomy Laboratory, 601 Campbell Hall, University of California, Berkeley, CA 94720; gbower,plambeck,bolatto,nate,jrg,imke@astro.berkeley.edu

${ }^{2}$ Institute for Astronomy, University of Hawaii, 2680 Woodlawn Dr., Honolulu, HI 96822; mliu@ifa.hawaii.edu

${ }^{3}$ Hubble Fellow

${ }^{4}$ Center for Space Research, Massachusetts Institute of Technology, Cambridge, MA 02139; fkb@space.mit.edu
} 


\section{Introduction}

The transient radio sky is mostly unexplored, especially at millimeter wavelengths. The few systematic radio searches for astronomical transients have been done at longer wavelengths, cover a small portion of the sky, or have low sensitivity (e.g., Langston et al. 2000, Hyman et al. 2002, Carilli et al. 2003), principally due to the lengthy observing necessary to conduct transient surveys with even the most sensitive of existing instruments. Many of the known radio transients, in fact, were first discovered at other wavelengths (e.g., Frail et al. 2003). Therefore, the population of transient millimeter wavelength sources is currently only poorly constrained. With the advent of new, more sensitive facilities (such as ALMA), we expect that discoveries of radio transients will be a common occurrence.

Radio stars are among the best studied transient sources at centimeter wavelengths (Güdel 2002). A wide range of stellar types have been shown to emit in the radio: brown dwarfs, T Tauri stars, dwarf stars, and evolved giants. The radio properties of young stellar objects (YSOs) have been studied in a variety of environments, particularly in the Orion nebula (Garay, Moran \& Reid 1987; Churchwell et al. 1987; Felli et al. 1993a, 1993b). These surveys show the existence of sub-arcsecond radio sources, many of which are variable and coincident with stars. Detection of radio circular polarization in YSOs has strengthened the case that flares from these stars originate in massive plasma ejections related to coronal magnetic field activity, qualitatively similar to those seen in the Sun (Feigelson, Carkman \& Wilking 1998, White, Pallavicini \& Kundu 1992). Although deep X-ray and infrared surveys of the Orion nebula have identified many variable sources (Feigelson et al. 2002; Hillenbrand \& Carpenter 2000; Carpenter, Hillenbrand \& Skrutskie 2001), the origins of magnetic activity in YSOs are still poorly understood (Feigelson et al. 2003).

We report here the serendipitous discovery of a millimeter-wave transient source in the Orion nebula cluster (Figure 1). The Berkeley-Illinois-Maryland Association Array (BIMA) detected the source at $86 \mathrm{GHz}$ on 2003 January 20, with a flux density that varied substantially on a timescale of hours (Bower, Plambeck \& Bolatto 2003). For a time the object became the brightest source in the cluster, with a flux density greater than those of the Becklin-Neugebauer Object (BN) and the massive young star IRc2. This detection was possible only because BIMA was in its highest resolution configuration, which provides a $0^{\prime \prime} .5$ synthesized beam and filters out the much stronger extended emission from dust, molecular lines, and the Orion HII region.

The source position, measured to an accuracy of $\pm 0.1^{\prime \prime}$ from the BIMA image, was found to be coincident with a variable centimeter-wave radio source (GMR-A; Garay et al. 1987, Felli et al. 1993a,b), a bright near infrared point source (number 573; Hillenbrand \& Carpenter 2000), and a variable X-ray source (number 297; Feigelson et al. 2002). These 
previous observations suggested that GMR-A is a YSO deeply obscured by the molecular cloud. New observations presented here support and expand this conclusion.

Follow-up observations with BIMA and the Very Large Array (VLA) showed that the source decayed within days of its outburst. GMR-A flared again on several occasions over the 70 days following the first flare, although never to the discovery intensity. The Nobeyama Radio Observatory (NRO) detection of GMR-A at 100 and $150 \mathrm{GHz}$ a few days after discovery (Nakanishi et al. 2003) found an inverted spectrum, indicating that the synchrotron selfabsorption frequency of the plasma was greater than $100 \mathrm{GHz}$. Circularly polarized emission was detected with the VLA at several epochs, indicating that the radio emission was caused by cyclotron radiation from mildly relativistic electrons in a strong magnetic field. Very Large Baseline Array (VLBA) observations within 10 days of the discovery showed that a significant fraction of the flux density of GMR-A was unresolved on the milliarcsecond scale.

By an extraordinary coincidence, the Chandra X-ray Observatory was in the midst of a very deep integration on the Orion nebula at the time of the BIMA discovery (Getman et al. 2003). The X-ray flux from GMR-A increased by a factor of $\sim 10$ approximately 2 days prior to the BIMA observation. To our knowledge, this is just the second simultaneous radio/mm and X-ray observation of a YSO (Feigelson et al. 1994) and the only simultaneous radio/mm and X-ray observation of a flaring YSO.

Infrared photometry at the Keck I 10-m and the Cerro Tololo Interamerican Observatory (CTIO) 4-m telescopes within days of discovery showed no variability with respect to the historical flux. Infrared spectroscopy with the Keck II 10-m telescope allowed a robust identification of the infrared source as a star of spectral type $\mathrm{K} 5 \mathrm{~V}$, with a fast rotation, a measurable Zeeman effect indicative of magnetic activity, and a weak Brackett $\gamma$ emission line. All evidence points to a strong magnetic outburst from a highly obscured young stellar object, probably a weak-line T Tauri star.

\section{Observations and Data Analysis}

This paper presents observations obtained at BIMA, the VLA, the VLBA, Keck, and the CTIO. Plots of the light curve including dates for infrared photometry, infrared spectroscopy and X-ray observations are shown in Figures 2, 3 and 4. In addition to the observations described below, we have included the NRO observations reported by Nakanishi et al. (2003) and the Chandra X-ray observations reported by Getman et al. (2003). Positions determined from various methods are listed in Table 1. Flux densities from BIMA, VLA, and near infrared observations are listed in Tables 2,3 , and 4 . We discuss each data set in detail 
below.

\subsection{BIMA Observations}

Continuum observations of the Orion nebula at $86 \mathrm{GHz}$ were made with the BIMA array on 2002 December 23 and 2003 January 20 as part of a long term project to track the proper motion of the Becklin Neugebauer Object relative to IRc2 (Plambeck et al. 1995). BIMA was in its A-configuration (Welch et al. 1996), which provides telescope spacings of up to $1.9 \mathrm{~km}$; the synthesized beam on Orion was $00^{\prime \prime} 9 \times 0$. .5 . The intense $\mathrm{v}=1 \mathrm{~J}=2 \rightarrow 1 \mathrm{SiO}$ maser associated with IRc2 was used as a phase reference for self-calibration.

Figure 1 shows the discovery image, from the 2003 January 20 data set. The map covers a $2.5 \times 2$ '.5 region and has not been corrected for attenuation by the 2.2 BIMA primary beam. The flaring object is conspicuous in the upper right quadrant. Note that emission from dust, molecular lines, and the Orion HII region is almost completely resolved out in this image. There is no sign of the flare source in the 2002 December 23 image, to a $3 \sigma$ upper limit of $6 \mathrm{mJy}$ (after correction for primary beam attenuation). BIMA observed this field also on 2003 January 11 and 14, during very poor weather. Maps generated from these data show $\mathrm{BN}$ and IRc2 but no sign of the flare source, to levels of 12 and $15 \mathrm{mJy}$, respectively.

Following the preset schedule, BIMA was reconfigured from the A-array into the Barray on 2003 January 21, so all follow-up observations of the flare were obtained with a $5.5 \times 2$. 5 synthesized beam. To exclude extended emission, only baselines longer than $20 k \lambda$ were used to make these maps. Fortunately, the flare source is far enough from the OrionKL nebula that confusion from dust or spectral line emission is unimportant at this angular resolution. Brief follow-up observations were conducted on 2003 January 23, 24, and 30 and 2003 February 4 and 6. GMR-A was detected only on 2003 February 6, with a flux density of $11 \pm 3 \mathrm{mJy}$.

\subsection{VLA Observations}

VLA observations were carried out on 19 occasions beginning 2003 January 22 (two days after the discovery observations) and ending 2003 March 29. Before 17 February 2003 intervals between observations ranged from one day to 5 days. Observations had durations ranging from 1 to 3.5 hours. They were conducted in the standard continuum mode with two polarizations and two IF bands of $50 \mathrm{MHz}$. Observing frequencies of 8.4, 15, 22 and 43 $\mathrm{GHz}$ were used, although not all bands were observed in each epoch. 
The data were analyzed in AIPS. The absolute flux density scale was set by observations of J0713+438. Following the VLA calibrator manual, we assume for J0713+438 $S_{8.4}=$ $1.14 \mathrm{Jy}, S_{15}=0.73 \mathrm{Jy}, S_{22}=0.55 \mathrm{Jy}$, and $S_{43}=0.29 \mathrm{Jy}$. These fluxes agree within $10 \%$ of measurements from 27 December 2002 and 8 February 2003 at 8.4, 22 and $43 \mathrm{GHz}$ (http://www.aoc.nrao.edu/ smyers/calibration/). Antenna pointing was stabilized using $\mathrm{X}$-band reference pointing on J0607-085. Phase, amplitude, and leakage term calibration were performed with observations of J0607-085. Since these observations are brief, the leakage term calibration cannot be regarded as providing accuracy in the linear polarization better than $1 \%$. Instrumental errors in the fractional circular polarization are $<1 \%$ (Bower et al. 2002).

The VLA was in the DnC and D configuration during these observations, providing a typical resolution of $3^{\prime \prime} \times 1^{\prime \prime}$ at $22 \mathrm{GHz}$. Because of the substantial extended flux associated with the Orion nebula, only visibilities on baselines longer than $20 k \lambda$ were used. The typical image rms noise in a 1-hour long observation was 10 mJy, 3 mJy, 1 mJy and 1 mJy at 8.4, 15, 22 and $43 \mathrm{GHz}$, respectively. Flux densities for GMR-A were measured by fitting for a point source at the phase center. GMR-A was never detected at $8.4 \mathrm{GHz}$ because confusion from extended emission in the $10^{\prime \prime}$ synthesized beam dominated the source. We also measured the flux density and the position of $\mathrm{BN}$ at $22 \mathrm{GHz}$ at each epoch. The stability of these quantities gives us confidence in the accuracy of our measurements for GMR-A. We find a mean flux density for the BN object at $22 \mathrm{GHz}$ of $12.9 \pm 0.1 \mathrm{mJy}$.

\subsection{VLBA Observations}

Observations with the VLBA were carried out on 2003 January 24 and 29 for 2 and 6 hours, respectively. Observing frequencies were 15 and $22 \mathrm{GHz}$ in both experiments. The observations were performed in a phase-referenced mode that allows detection of a weak source. The reference source used was J0541-0541, which is 1.6 degrees away from GMR-A. We assumed a position $\alpha=05: 41: 38.0833740$ and $\delta=-05: 41: 49.428471$ (J2000) for J05410541. A cycle time (that includes a calibrator observation, slew to the source, a source observation and slew back to the calibrator) of 40 seconds was used in the first epoch. Cycle times of 60 and 120 seconds were used at 15 and $22 \mathrm{GHz}$ in the second epoch, respectively. Fringe solutions were determined for most antennas in both experiments using the calibrator

J0541-0541. One iteration of self-calibration amplitude solutions were also computed for J0541-0541, which substantially improved the image quality. These solutions were applied to GMR-A.

A large field was imaged and the source was clearly identified in the second epoch at 
15 and $22 \mathrm{GHz} \sim 40$ mas from the field center (Figure 5). Exact coordinates are given in Table 1. The reported errors in the position are $\sim 20 \mu$ as in right ascension and $\sim 40 \mu$ as in declination. These are statistical errors. Following Reid et al. (1999), we estimate that systematic errors due to miscalibration of the opacity are $\sim 100 \mu$ as.

During the second VLBA epoch, the integrated flux densities measured for GMR-A were $11.2 \pm 0.8 \mathrm{mJy}$ and $16.7 \pm 1.3 \mathrm{mJy}$ at 15 and $22 \mathrm{GHz}$, respectively. These are substantially less than the VLA fluxes measured 1 day later, of $32.4 \pm 1.7 \mathrm{mJy}$ and $30.1 \pm 0.8 \mathrm{mJy}$ at 15 and $22 \mathrm{GHz}$. The source appears point-like in the $22 \mathrm{GHz}$ image with a resolution of $1.4 \times 0.95$ mas: its maximum deconvolved size is 0.7 mas. GMR-A is slightly extended in the $15 \mathrm{GHz}$ image, which has a resolution of $2.0 \pm 0.60$ mas. At this frequency the source is equally well-modeled as a circular Gaussian of deconvolved size 0.9 mas, or as two point-like components separated by 0.8 mas and of flux density $8.2 \pm 0.3$ and $3.3 \pm 0.3 \mathrm{mJy}$.

No source was detected at either 15 or $22 \mathrm{GHz}$ in the first VLBA epoch at a level of $5 \sigma$. The rms noise in the 15 and $22 \mathrm{GHz}$ images is $1.3 \mathrm{mJy}$ and $1.6 \mathrm{mJy}$, respectively. The VLA fluxes on the same day were $18.5 \pm 1.7 \mathrm{mJy}$ and $23.7 \pm 0.6 \mathrm{mJy}$, respectively. A number of factors may contribute to the nondetection of GMR-A in the first epoch, and to the discrepancy in flux density in the second epoch. Instrumental errors due to phase decorrelation and amplitude calibration may contribute substantially. Additionally, we know that the source can vary significantly on a time scale of hours. Another plausible explanation

is that some of the flux is in a low-surface brightness "halo" which is resolved out by the VLBA. We discuss this possibility, which is supported by earlier VLA observations, in more detail in $§ 3.1$.

\subsection{Near Infrared Photometry}

Observations of GMR-A were made on 2003 January 22 at 6 UT with the Keck I 10-m telescope on Mauna Kea, Hawaii, and on 2003 January 24 at 1 UT with the Cerro Tololo Interamerican Observatory Blanco 4-m telescope at Tololo, Chile.

The Keck observations used the facility near-infrared camera (NIRC; Matthews \& Soifer 1994), which is equipped with a $256 \times 256$ pixel Santa Barbara Research Corp. InSb array, with a pixel size of $0.151^{\prime \prime}$. The images were taken at an airmass of 1.26 , and under seeing conditions of $0.9^{\prime \prime}$. We flat-fielded the data according to standard procedures, and replaced bad pixels with the median of surrounding pixels. The absolute calibration of the images was set by observing the IR standard star SJ9118 (Persson et al. 1998) at a similar airmass (1.28). Photometry in $\mathrm{J}, \mathrm{H}$, and $\mathrm{K}_{S}$ bands was obtained for GMR-A and two other stars 
in the field. We simply integrated the intensity over the star and subtracted the average background emission. Corrections from $\mathrm{K}_{S}$ to $\mathrm{K}$ band were less than $0.1 \mathrm{mag}$.

We obtained additional IR photometry from the Blanco 4-m Telescope at the Cerro Tololo Interamerican Observatory with the facility wide-field camera Infrared Side Port Imager (ISPI; Probst et al. 2003). The instrument uses a Rockwell $2048 \times 2048$ pixel HgCdTe detector with a $10.2^{\prime}$ field of view. Conditions were photometric with seeing of $1.6^{\prime \prime}$ FWHM. GMR-A was too bright at $K_{S}$-band to be observed in the shortest possible integration time, so we de-focused the telescope in order to obtain unsaturated images for this filter. We observed the standard star SJ 9116 from Persson et al. (1998) for photometric calibration, also with the telescope defocused for $K_{S}$-band. The data were reduced in a standard fashion. We subtracted an average bias frame from the images. We constructed flat fields from images of the lamp-illuminated interior of the dome. The individual images were registered via cross-correlation and stacked to form a final mosaic. Aperture photometry was used to measure the object and standard star fluxes.

Comparison between the Keck and CTIO results for GMR-A and two other field stars (Table 4) suggests that the photometric accuracy is on the order of 0.1 mag.

\subsection{Near Infrared Spectroscopy}

We observed GMR-A with the Keck II 10-m telescope on 2003 February 6, using the facility near-infrared echelle spectrometer (NIRSPEC; McLean et al. 1998). We obtained high-resolution $(R \sim 21,000)$, cross-dispersed spectra in the wavelength range $2.10-2.40 \mu \mathrm{m}$ using the NIRSPEC-7 order-sorting filter. The star was imaged in two nods along the $0^{\prime \prime} 432$ $\times 24^{\prime \prime}$ slit, separated by $12^{\prime \prime}$ (spatial resolution was seeing limited at $\sim 1^{\prime \prime}$ ). Each integration was 300 seconds, providing a total time of 600 seconds on the star and a signal-to-noise ratio of approximately 160 . The spectra were dark subtracted, flat-fielded, and corrected for cosmic rays and bad pixels. The curved echelle orders were then rectified onto an orthogonal slit-position/wavelength grid based on a wavelength solution from sky $(\mathrm{OH})$ emission lines and $\mathrm{He} / \mathrm{Ar} / \mathrm{Ne} / \mathrm{Kr}$ arc lamps. Each pixel in the grid has a width of $\delta \lambda=0.031 \mathrm{~nm}$. We subtracted sky emission by taking the difference of the two nods and then fitting third-order polynomials to the $2 \mathrm{D}$ spectra column-by-column to remove any temporal variation.

The star's spectrum was extracted using a Gaussian weighting function matched to the wavelength-integrated profile of each echelle order. To correct for atmospheric absorption, we observed a B0V star, HD 36512; both GMR-A and the calibration star were observed at an airmass of $\sim 1.2$. To account for photospheric absorption features and continuum 
slope, the calibration star's spectrum was divided by a spline function fit. The resulting atmospheric absorption spectrum was then divided into the GMR-A spectra.

It is difficult to separate stellar from nebular emission for GMR-A. $\mathrm{Br} \gamma$ emission at $2.166 \mu \mathrm{m}$ fills the slit with a velocity width of $29.7 \mathrm{~km} \mathrm{~s}^{-1}$ and an equivalent width of $\sim 6 \AA$. Subtraction of the mean off-source spectrum from that of GMR-A reveals a weak emission line with $\mathrm{FWHM} \sim 23 \mathrm{~km} \mathrm{~s}^{-1}$ and $\mathrm{EW} \sim 0.8 \AA$.

\section{Discussion}

\subsection{Radio, Infrared, and X-ray Properties}

The mm-wavelength flare source has counterparts at radio, infrared and X-ray wavelengths. The object was first identified (as "source A") by Garay et al. (1987) from VLA observations obtained in 1981; its flux densities were $10.0 \pm 1.0 \mathrm{mJy}$ and $11.0 \pm 1.0 \mathrm{mJy}$ at 5 and $15 \mathrm{GHz}$, respectively. Later VLA observations by Felli et al. (1993a) gave flux densities of $5.5 \pm 0.5 \mathrm{mJy}$ and $5.4 \pm 0.3 \mathrm{mJy}$ at 1.4 and $15 \mathrm{GHz}$. Both studies found evidence that the source was slightly extended; Felli et al. found a deconvolved size of $0.19 \times 0.14$ arcsec. From observations at 13 epochs over an 8-month period in 1990, Felli et al. (1993b) found that the flux density of GMR-A was highly variable at 5 and $15 \mathrm{GHz}$, with a flat spectrum. Our VLBA observations, in combination with the minimum flux density in the VLA lightcurve, suggest that GMR-A consists of a constant, extended, flat spectrum source with a flux density $\sim 5$ mJy which is resolved out by the VLBA and a variable, compact source that is detected by the VLBA.

As shown in Figure 6, the radio spectral index was highly variable in the weeks following the initial BIMA detection. On 22 January, 2 days after the flare was detected at BIMA, the emission had a flat spectrum between 22 and $43 \mathrm{GHz}\left(\alpha \approx-0.1\right.$ for $\left.S \propto \nu^{\alpha}\right)$, but appeared to cut off sharply below $22 \mathrm{GHz}$. Two days later, as the first outburst cooled, the spectrum remained peaked at roughly $22 \mathrm{GHz}$, with a higher frequency spectral index of -0.5 . One day later, on 25 January, Nobeyama observations (Nakanishi et al. 2003) detected the source at 98 and $147 \mathrm{GHz}$; the spectrum was inverted $(\alpha=1.4)$, implying that the synchrotron

self-absorption frequency of the plasma was greater than $\sim 100 \mathrm{GHz}$. On 7 February the source appeared to have a flat spectrum from 15 to $86 \mathrm{GHz}$. Much later, on 27 March, the spectral index is -0.7 , although the absence of an $86 \mathrm{GHz}$ observation makes this steep spectrum somewhat uncertain.

Circular polarization is clearly detected in many epochs (Table 3). When either the circular polarization or the total intensity is not detected at the $2-\sigma$ level, we report an 
upper limit to the absolute value of the circular polarization. The mean fractional circular polarization (measured as $V / I$ ) is $-3.4 \pm 0.4 \%,-3.9 \pm 0.2 \%$ and $-6.6 \pm 0.6 \%$ at 15,22 and $43 \mathrm{GHz}$, respectively. Nevertheless, there is clear time variability in the circular polarization: in particular, we did not detect circular polarization near the peak of the flare. The emission is never linearly polarized. We searched for linear polarization but did not detect it in any epoch with limits $\sim 1 \%$.

The near infrared counterpart of GMR-A is bright, and not variable. GMR-A is positionally coincident with source \#573 in the infrared survey of Hillenbrand and Carpenter (2000). Our infrared photometry gives H and K magnitudes consistent with those measured by Hillenbrand and Carpenter (Table 4). Furthermore, the intensive survey for variability in the Orion nebula by Carpenter, Hillenbrand \& Skrutskie (2001) did not detect this object as significantly variable.

In contrast, the X-ray counterpart of GMR-A is characterized as a flare source by Feigelson et al. (2002); it is substantially variable on a timescale of less than 12 hours as well as over six months. The spectrum is consistent with an intrinsic $\mathrm{X}$-ray luminosity $L_{x}=10^{31.7} \mathrm{erg} \mathrm{s}^{-1}$ attenuated by a gas column density $N_{H}=10^{22.6} \mathrm{~cm}^{-2}$. This luminosity ranks it among the brightest $10 \%$ of the X-ray sources in the Orion nebula. Getman et al. (2003) reported a significant $\mathrm{X}$-ray flare $(\times 10)$ at its position. Their preliminary light curve shows the flare beginning $\sim 2$ days before the detected millimeter wave flare, and continued through the time of the radio flare. Of course, we are not able to accurately determine the onset of the flare at millimeter wavelengths because of the scarcity of observations. The $\mathrm{X}$-ray flux was declining at the time of the millimeter flare. Modeling of the X-ray spectrum by Getman et al. during the flare indicates an absorption column density comparable to that found earlier.

\subsection{Variability Timescale of the Radio Emission}

To study the flux variability during the onset of the radio flare, we broke up the 8-hour long visibility data obtained by BIMA on the night of 2003 January 20 into 1-hour blocks. The flare brightened by a factor of $\sim 4$ to 8 during the track (Figure 2), while the fluxes of IRc2 and BN remained stable. The flux density of the flare source was fit in each interval after subtracting the contributions from point sources at the positions of IRc2 and BN with flux densities of 45 and 68 mJy respectively. Because the BIMA receivers are sensitive to linear polarization in the vertical direction, a concern is that the observed variability may not be intrinsic, but introduced by linearly polarized emission combined with changes in the parallactic angle during the night. The parallactic angle changed from $-38^{\circ}$ to $+45^{\circ}$ 
during the observations. If this were the cause of the variability, the emission would have to be $>45 \%$ linearly polarized, which is highly unusual. A broad range of position angles and polarization fractions were used to model the light curve, obtaining a minimum reduced $\chi^{2} \sim 17$, suggesting that linearly polarized emission cannot reproduce the observations. Furthermore, linear polarization is undetected in all subsequent VLA observations. It is very unlikely that the $86 \mathrm{GHz}$ flux is initially strongly polarized while two days later the 43 $\mathrm{GHz}$ flux is not. This fact, together with the poor quality of the fit, strongly argue that the observed $86 \mathrm{GHz}$ lightcurve is due to intrinsic variability and not to polarization.

We searched for short-term variations in the VLA data, as well. Most of the observations were split into two or more blocks probing timescales that range from 0.5 to 1.5 hour. There was no evidence for significant variability on these timescales.

\subsection{Identification of GMR-A as a Young Stellar Object}

To determine the spectral type of GMR-A, we compared its near-IR spectrum with the medium-resolution $K$-band spectral atlas of Wallace \& Hinkle (1997). The relative strengths of the $\mathrm{Al}$ and $\mathrm{Mg}$ lines near $2.11 \mu \mathrm{m}$, as well as the relative strengths of the $\mathrm{Ti}$ and Si lines near $2.18 \mu \mathrm{m}$, indicate a spectral type of K5. The shallow CO bandheads are consistent with a dwarf star. On this basis, we identify GMR-A as a spectral type K5V.

The ${ }^{12} \mathrm{CO}(\mathrm{v}=0 \rightarrow 2)$ rovibrational band (extending redward from the bandhead at $2.29 \mu \mathrm{m}$ ) allows us to measure the radial and rotational velocities of the star, $v_{l s r}$ and $v \sin i$, respectively. As a template for our rotational model, we used a high-resolution FTS sunspot umbral spectrum (Wallace \& Hinkle 2001) with $T_{\text {eff }}=4100 \mathrm{~K}$. We convolved the sunspot spectrum with a rotational broadening profile from Gray (1976), and convolved the result with the measured profile of an $\mathrm{OH}$ line observed with NIRSPEC to account for the instrumental resolution. Iterating over a range of rotational velocities, we find $v \sin i=23 \pm 2$ $\mathrm{km} \mathrm{s}^{-1}$ and $v_{l s r}=-4 \pm 5 \mathrm{~km} \mathrm{~s}^{-1}$. The quoted error in $v \sin i$ reflects the broad nature of the minimum in the residuals for models near $23 \mathrm{~km} \mathrm{~s}^{-1}$. Our determination of $v_{l s r}$ suggests that GMR-A is not embedded in the molecular cloud, for which the radial velocity from $\mathrm{NH}_{3}$ measurements is $v_{l s r}=9 \pm 1 \mathrm{~km} \mathrm{~s}^{-1}$ (Batrla et al. 1983), but rather lies behind it.

Despite the difficulties in subtracting the background emission from the nebula, the observed $\operatorname{Br} \gamma$ emission is clearly substantially weaker and narrower than is typical in classical T Tauri stars (Folha \& Emerson 2001), indicating that this is probably a weak line T Tauri star (WTTS). This designation is also consistent with strong radio variability, as WTTS are the most luminous and most variable of radio YSOs. 
Taken together, the X-ray data, IR photometry, and IR spectroscopy point to a clear identification of GMR-A as a YSO inside, or perhaps behind, a molecular cloud. However, the identification of GMR-A as a $\mathrm{K} 5 \mathrm{~V}$ is not consistent with the high luminosity implied by the very bright $\mathrm{K}$ magnitude and the large extinction. We discuss here the different scenarios that might resolve this, including different degrees of extinction, the presence of an infrared excess, and classification as a K5III star, but none of these are fully satisfactory. In the simplest approach, we use the X-ray absorption column depth and $R_{V}=3.1$ to find the extinction $A_{V}=21.4$ which implies $A_{K}=2.4$ mag (Rieke \& Lebofsky 1985; Binney \& Merrifield 1998). Using color and bolometric corrections appropriate for K5V stars, we find $L \sim 30 L_{\odot}$. But this result is almost certainly wrong for two reasons: (1), this luminosity exceeds the luminosity of the stellar birth line, which is $<10 L_{\odot}$ at this effective temperature (Palla \& Stahler 1999); and (2), the photospheric H-K color of 1.0 after applying reddening correction is not appropriate for a K5V star. Assuming a photospheric color $\mathrm{H}-\mathrm{K}=0.1$, which is the case for $\mathrm{K} 5 \mathrm{~V}$ stars, we find $A_{V}=35$ and $A_{K}=3.9$. A similar extinction is obtained with examination of the $\mathrm{J}-\mathrm{H}$ and $\mathrm{H}-\mathrm{K}$ colors (e.g., Haisch, Lada \& Lada 2000). While this satisfies the spectroscopic constraints, it produces a luminosity of $100 L_{\odot}$, again in excess of the stellar birth line. This inconsistency suggests an excess of infrared emission possibly due to a disk. Assuming the reddening calculated by the $\mathrm{X}$-ray absorption column depth $\left(A_{K}=2.4\right)$ and a photospheric color $\mathrm{H}-\mathrm{K}=0.1$, then we find an $\mathrm{H}-\mathrm{K}$ excess of 0.9 mag and a K-band excess of $1.7 \mathrm{mag}$ (Hillenbrand \& Carpenter 2000). This reduces the bolometric luminosity to $6 L_{\odot}$. While this satisfies the stellar birth line luminosity limit and the colors for these stellar classes, the presence of a strong infrared excess is more consistent with a classical T Tauri star than with a WTTS, although some WTTS do have an IR excess (Strom et al. 1989). Considering the same scenarios for K5 giant or supergiant stars leads to the same problems.

Could GMR-A be something other than a T Tauri star? A possibility is that the flaring source active in radio and $\mathrm{X}$-rays is not the object dominating the IR luminosity, hence the lack of infrared activity. There is some precedent for faint companion stars producing intense variable radio emission: astrometric radio and optical measurements of $\theta^{1}$ Ori A have shown that the object responsible for radio flaring is actually part of a triple system (Garrington et al. 2002). The "active" companion, however, would have to be several magnitudes fainter than the main K5V star even during its flaring state, to account for its non detection in the IR spectrum. Adaptive optics observations in the infrared would be necessary to identify any faint companions (e.g., Duchêne et al. 2003). Other potential radio transient source types are brown dwarfs and late-type main sequence stars (e.g., Berger 2002). These sources, however, are too faint by many orders of magnitude to account for the quiescent or flaring radio emission from GMR-A. Evolved stars such as RS Cvn binaries are unlikely to be 
associated with the Orion complex. In the following section we show that the magnetic field measurements also support the conclusion that GMR-A is a T Tauri object.

\subsection{Zeeman Measurement of Magnetic Field Strength}

We used the echelle infrared spectrum to search for Zeeman broadening of lines in the stellar photosphere, which would indicate the presence of a strong magnetic field. We selected the strong TiI lines at $\lambda=2.17886 \mu \mathrm{m}$ and $\lambda=2.19033 \mu \mathrm{m}$ present in echelle order 35 in our spectra to carry out this study. These transitions have spectroscopic terms $\left(3 \mathrm{~d}^{35} \mathrm{P}_{3} \rightarrow 4 \mathrm{p}^{15} \mathrm{D}_{4}\right)$ and $\left(3 \mathrm{~d}^{35} \mathrm{P}_{2} \rightarrow 4 \mathrm{p}^{15} \mathrm{D}_{3}\right)$ respectively, resulting in very similar effective Landé $g$-factors, 1.667 and 1.833. Because the $g_{L}$ factors of the upper and lower energy levels in either transition are not precisely equal, these lines formally exhibit the "anomalous" Zeeman effect and they are broken up into seven components by the magnetic field. In practice, since the Landé factors are almost equal, the emission is separated into approximately 3 equal components.

We modeled the observed emission using 3 spectral lines with the shape of the intrinsic instrumental profile convolved with the rotational profile corresponding to the measured value of $v \sin i=23 \mathrm{~km} \mathrm{~s}^{-1}$. Thus, we assume that the only sources of line broadening are instrumental, rotational, and Zeeman, an assumption that seems justified in view of the large $v \sin i$. These models were computed for a grid of Zeeman splittings, $\Delta \lambda$, and line amplitudes. We determined the best model by evaluating the reduced $\chi^{2}$ of the residuals. The error was estimated by finding the values of the parameters that increased the reduced $\chi^{2}$ by one. Figure 7 shows the results of the fit for the $\lambda=2.17886 \mu \mathrm{m}$ transition. The measured Zeeman splittings are $\Delta \lambda=0.14 \pm 0.07 \mathrm{~nm}\left(\chi^{2}=1.4\right)$ and $\Delta \lambda=0.09 \pm 0.05 \mathrm{~nm}\left(\chi^{2}=1.7\right)$, corresponding to magnetic fields $3.8 \pm 2 \mathrm{kG}$ and $2.2 \pm 1.1 \mathrm{kG}$. Thus, our best estimate of the photospheric magnetic field traced by the TiI lines is $2.6 \pm 1.0 \mathrm{kG}$. Observations of T Tauri objects find similar magnetic field strengths (e.g., Johns-Krull, Valenti \& Koresko 1999).

\subsection{Radio Emission Mechanism}

Several lines of evidence indicate that the radio emission mechanism is nonthermal: the compactness of the emission detected using the VLBA, the presence of circular polarization, the strongly variable spectral index, and the rapid rise and decay time of the total intensity. We can set a lower limit to the brightness temperature based on the flux measured by the VLBA observations. We find $T_{b} \gtrsim 5 \times 10^{7} \mathrm{~K}$ and $T_{b} \gtrsim 3 \times 10^{7} \mathrm{~K}$ at 15 and $22 \mathrm{GHz}$. Electrons 
in equilibrium with this temperature will have velocity $\gtrsim 0.07 c$, or an electron Lorentz factor $\gamma_{e} \gtrsim 1.003$.

The presence of high fractional circular polarization rules out thermal bremsstrahlung and synchrotron radiation as emission mechanisms, and indicates that the dominant emission is cyclotron radiation generated by mildly relativistic electrons in the presence of strong magnetic fields (e.g., Güdel 2002). The observed mean fractional circular polarization in the VLA bands is $\sim-3$ to $-7 \%$, with peak values greater than $-10 \%$. The absence of linear polarization also argues against synchrotron emission, although very tangled magnetic fields or a high rotation measure due to an intervening dense, magnetized plasma could depolarize a synchrotron source.

The radio emission probably originates in coronal magnetic fields, which are an order of magnitude weaker than the photospheric magnetic field strength determined in the previous section. Assuming a field strength for the radio emission of $100 \mathrm{G}$, the corresponding electron gyro-frequency is $0.5 \gamma_{e}^{-1} \mathrm{GHz}$. The lack of a very steep fall-off at high frequencies, characteristic of a single temperature thermal cyclotron emitter, leads us to conclude that the emission originates from a power-law distribution of electrons with low energy. A power-law distribution of energies with index $\delta=1.5$ yields an optically-thin spectrum with $\alpha=-0.1$ above a peak frequency of $22 \mathrm{GHz}$. The characteristic size of the emitting region for the assumed magnetic field is $2 \times 10^{11} \mathrm{~cm}$. For comparison, models indicate that the radius of a YSO with $L=3.5 L_{\odot}$ and $T_{\text {eff }}=4100 \mathrm{~K}$ is $\sim 4 \times 10^{11} \mathrm{~cm}$, suggesting that the cyclotron emission is a global phenomenon for the star.

A significant anomaly in this picture is the absence of circular polarization in the first VLA observation on January 22, 2 days after the millimeter wavelength flare. A possible mechanism to initially supress circular polarization is to invoke an initial outburst of relativistic electrons, leading to synchrotron rather than cyclotron emission. Rapid cooling through expansion or X-ray radiation would then drive the radio source into the cyclotron regime. In this scheme one expects that the early radiation would be linearly polarized. We have seen that VLA observations on day 22 failed to show any linear polarization. As discussed above, however, there are mechanisms that could significantly depolarize a synchrotron source. An alternative explanation for the initial lack of circular polarization assumes the existence of two oppositely polarized sources associated with the star. Imaging of some flaring radio stars has shown such oppositely polarized lobes (Mutel et al. 1998). Circular polarization disappears when these lobes are equally balanced and appears when one lobe comes to dominate the emission. Without more detailed modeling and/or imaging of the source we cannot discriminate between these two possibilities.

The source reflared several times during the course of the follow-up observations, albeit 
never to the level of the discovery data. In fact, the light curve appears to consist of multiple flares spread over $\sim 70$ days, with individual flares having a rise and decay timescale of days or shorter. During the BIMA discovery, we measure a rise time of $\sim 1$ hour. Since this is the duration of most of the VLA observations, we cannot clearly discern whether there is a similar variability timescale in those data. The timescales for radiation losses for a cyclotron source are proportional to $B^{-2}$, and are $\sim 1$ day for $B=100 \mathrm{G}$, which is comparable to what is observed.

\subsection{The Radio to $\mathrm{X}$-ray Luminosity Correlation}

The luminosity of this flare is comparable to that of the brightest stellar radio flare ever detected. The peak luminosity at $86 \mathrm{GHz}$ during the flare was $4 \times 10^{19} \mathrm{erg} \mathrm{s}^{-1} \mathrm{~Hz}^{-1}$, only $50 \%$ less than the peak flare of the current record-holder, the FK Com-type giant star HD32918 (Slee et al. 1987, Bunton et al. 1989). The luminosity of the GMR-A flare, however, was more than an order of magnitude higher than detected for any YSO. Typical maximum radio luminosities for YSOs are $\sim 10^{18} \mathrm{erg} \mathrm{s}^{-1} \mathrm{~Hz}^{-1}$ (Güdel 2002). Since GMR-A flared at millimeter wavelengths, its total luminosity was $L_{\nu} \sim 3 \times 10^{30} \mathrm{erg} \mathrm{s}^{-1}$, about two orders of magnitude higher than that of centimeter wavelength sources.

The ratios of $\mathrm{X}$-ray and radio emission for GMR-A during both its quiescent and flaring states approximately follow the correlation found for active stars and solar flares (Güdel 2002)

$$
\frac{L_{x}}{L_{r}} \approx 10^{15 \pm 1}[H z]
$$

This correlation was derived from radio observations at centimeter wavelengths (typically $8 \mathrm{GHz}$ ): the precise value of the coefficient is unknown for measurements at millimeter wavelengths. To compute the luminosities, we adopted the quiescent, absorption-corrected X-ray luminosity $L_{x}=10^{31.7} \mathrm{erg} \mathrm{s}^{-1}$ measured by Chandra. During the flaring state the Chandra observations show the count rate increasing by a factor $\sim 10$, leading to a prediction of $L_{r} \approx 5 \times 10^{18 \pm 1} \mathrm{erg} \mathrm{s}^{-1} \mathrm{~Hz}^{-1}$, very similar to that observed at $86 \mathrm{GHz}$. These data support the conclusion that the flare is caused by magnetic activity common to other radio stars.

\subsection{Rate of Flaring Activity in the Orion Nebula}

We can parametrize the rate of millimeter wavelength flares in the Orion nebula as

$$
N\left(S>S_{0}\right)=A\left(\frac{S_{0}}{100 \mathrm{mJy}}\right)^{-\alpha} .
$$


Nonthermal activity often follows a power-law index $\alpha=1$. This is the first flare brighter than 100 mJy detected by BIMA in $\sim 40$ observations of the Orion nebula, setting $A=$ 0.01-0.1 day ${ }^{-1}$. This value of $A$ is consistent with the 22 and $43 \mathrm{GHz}$ observations described in this paper, as well as the $15 \mathrm{GHz}$ dataset of Felli et al. (1993b). Both of these datasets show that GMR-A has a flux density of 10 mJy about 10 times as frequently as it has a flux density $\sim 100$ mJy. Following Equation 2, observations of Orion at a 0.1 mJy detection threshhold will find $\sim 10-100$ flares from YSOs per observation. In fact, the monitoring campaign of Felli et al. (1993b), with a sensitivity ranging between 0.2 and 1.2 mJy, identified 13 variable sources. The proposed Atacama Large Millimeter Array will achieve a sensitivity of 0.1 mJy or better in a 1 minute integration (http://www.alma.nrao.edu). Thus, a short ALMA observation with a sensitivity of $10 \mu \mathrm{Jy}$ may find $\sim 100-1000$ flares from YSOs, which is an appreciable fraction of the total number of objects in the region.

The same argument can be made using the $\mathrm{X}$-ray luminosity function. With a radio sensitivity of $3 \times 10^{15} \mathrm{erg} \mathrm{s}^{-1} \mathrm{~Hz}^{-1}$ (equivalent to $10 \mu \mathrm{Jy}$ ), the $L_{x} / L_{r}$ correlation predicts that we will detect all stars with $L_{x}>10^{30.5 \pm 1}$. This is consistent with the mean $\log L_{x}=29.4 \pm 0.7$ of the Chandra sample, suggesting that we would detect half of all X-ray objects in the Orion nebula (Feigelson et al. 2002). Approximately half of the 1075 objects in the Chandra sample are identified as variable on short and/or long timescales, indicating again that ALMA can expect to find hundreds of variable objects in a few hours.

Variable sources violate the assumption of a constant sky that underlies synthesis imaging. The potentially large number of variable sources in the Orion nebula and other starforming regions introduces a complication not yet considered for imaging with ALMA. If not adequately accounted for, these variable sources will introduce a dynamic range limit to images. Consider a source with a flare of flux density $S_{\max }$ over the quiescent flux density of $S_{q}$ that lasts for $t_{\max }<<t_{o b s}$, where $t_{o b s}$ is the total length of the observation. An array will have a peak fractional sidelobe level $\eta_{\max }$ that is a function of array configuration, source position and $t_{\max }$. To first order, this will introduce an imaging error $\eta_{\max } S_{\max }$ after deconvolution (e.g., using CLEAN). For ALMA, $\eta \sim 2 \%$ for observations of length $>1$ hour (e.g., Boone 2002). Thus, a brief $1 \mathrm{mJy}$ flare will introduce an error $>20 \mu \mathrm{Jy}$ in an image that could have a theoretical rms of $10 \mu \mathrm{Jy}$. Multiple sources and multiple flares complicate this picture but are unlikely to reduce the error. The solution, in principle, is to image and deconvolve on a timescale comparable to the flaring timescale. 


\section{Conclusions}

We have described here the serendipitous discovery and subsequent follow-up observations of a flaring millimeter wavelength source in the Orion star-forming cluster. The source appears to be a weak-line T Tauri star highly obscured by dusty material. Although previously known as a variable cm radio source (GMR-A), the magnitude of the flare and the measurement of its spectrum to millimeter wavelengths are novel. This is one of the most luminous radio flares observed, and the most luminous flare from a YSO. Future observations with greater sensitivity can be expected to detect many more of these objects.

The discovery of a compact source, detectable by the VLBA, in the Orion nebula raises the possibility of the measurement of trigonometric parallax to this star-forming region. It would be the most accurate distance measurement for Orion, achieving a parallactic error $\sim 100 \mu$ as equivalent to a distance error of $\sim 2 \%$ with only a few measurements. If the quiescent source is detectable, or if the source flares again, then two more observations are sufficient to separate the proper motion and parallax of this source. Monitoring and detection of other transient sources in this region and other galactic star-forming regions could significantly reduce the uncertainty in key star-forming parameters. A statistical approach is possible through VLBI astrometry of the $>10$ other nonthermal sources in Orion previously identified by Felli et al. (1993a). Finally, this may allow a 3-dimensional probe of the positions and velocities in the star forming cluster, giving vital clues to its history.

The BIMA array is operated by the Berkeley-Illinois-Maryland Association under funding from the National Science Foundation. The National Radio Astronomy Observatory is a facility of the National Science Foundation operated under cooperative agreement by Associated Universities, Inc. We thank Barry Clark for quickly and frequently scheduling follow-up VLA and VLBA observations of this source. The Keck data presented in this paper were obtained at the W. M. Keck Observatory, which is operated as a scientific partnership among the California Institute of Technology, the University of California and the National Aeronautics and Space Administration. The Observatory was made possible by the generous financial support of the W. M. Keck Foundation. The authors wish to recognize and acknowledge the very significant cultural role and reverence that the summit of Mauna Kea has always had within the indigenous Hawaiian community. We are most fortunate to have the opportunity to conduct observations from this mountain. It is a pleasure to thank Ron Probst, Nicole van der Bliek, and Angel Guerra for assistance with the CTIO observations. M. Liu is grateful for research support from the Beatrice Watson Parrent Fellowship at the University of Hawaii, the AAS Small Research Grants program, and NASA grant HST-HF- 
01152.01-A. We also thank Eric Feigelson, Konstantin Getman and their collaborators for promptly sharing Chandra data on this object, as well as their insights.

\section{References}

Batrla, W., Wilson, T.L., Bastien, P. \& Ruf, K., 1983, A\&A, 128, 279

Berger, E., 2002, ApJ, 572, 503

Binney, J. \& Merrifield, M., 1998, Galactic Astronomy, Princeton University Press, Princeton

Boone, F., 2002, ALMA Memos \#400

Bower, G.C., Falcke, H., Sault, R.J. \& Backer, D.C., 2002, ApJ, 571, 843

Bower, G.C., Plambeck, R.L. \& Bolatto, A., 2003, IAUC, 8055

Bunton, J. D., Large, M. I., Slee, O. B., Stewart, R. T., Robinson, R. D., \& Thatcher, J. D., 1989, PASA, 8, 127

Carilli, C., Ivison, R. \& Frail, D.A., 2003, AJ, in press

Carpenter, J.M., Hillenbrand, L.A. \& Skrutskie, M.F., 2001, AJ, 121, 3160

Churchwell, E., Felli, M., Wood, D.O.S., \& Massi, M. 1987, ApJ, 321, 516

Duchêne, G., Ghez, A.M., McCabe, C. \& Weinberger, A.J., 2003, ApJ, 592, 288

Feigelson, E.D. et al. , 1994, ApJ, 432, 373

Feigelson, E.D., Carkner, L. \& Wilking, B.A., 1998, ApJ, 494, L215

Feigelson, E.D. et al. , 2002, ApJ, 574, 258

Feigelson, E.D. et al. , 2003, ApJ, 584, 911

Felli, M., Churchwell, E., Wilson, T.L. \& Taylor, G.B., 1993a, A\&AS, 98, 137

Felli, M., Taylor, G.B., Catarzi, M., Churchwell, E. \& Kurtz, S., 1993b, A\&AS, 101, 127

Folha, D.F.M. \& Emerson, J.P., 2001, A\&A, 365, 90

Frail, D.A., Kulkarni, S.R., Berger, E. \& Wieringa, M.H., 2003, AJ, 125, 2299

Garay, G., Moran, J.M. \& Reid, M.J., 1987, ApJ, 314, 535

Garrington, S. et al. , 2002, 6th Euro. VLBI Net. Symp., Ros, E., Porcas, R.W., Lobanov, A.P \& Zensus, J.A., eds.

Getman, K.V., Feigelson, E.D., Garmire, G., Murray, S.S., Harnden, F.R.,Jr. , 2003, IAUC, 8068

Gray, D.F., 1976, Wiley-Interscience, New York

Güdel, M., 2002, ARA\&A, 40, 217

Haisch, K.E., Lada, E.A. \& Lada, C.J., 2000, AJ, 120, 1396

Hillenbrand, L.A. \& Carpenter, J.M., 2000, ApJ, 540, 236

Hyman, S. D., Lazio, T. J. W., Kassim, N. E. \& Bartleson, A. L., 2002, AJ, 123, 1497

Johns-Krull, C.M., Valenti, J.A. \& Koresko, C., 1999, ApJ, 516, 900 
Langston, G., Minter, A., D’Addario, L., Eberhardt, K., Koski, K. \& Zuber, J., 2000, AJ, 119,2801

Matthews, K., and B.T. Soifer, 1994, in Infrared Arrays in Astronomy: The Next Generation (I. S. McClean, Ed.), pp. 239-246, Kluwer, Dordrecht

McLean, I.S. et al. , 1998, SPIE, 3354, 566

Mutel, R.L., Molnra, L.A., Waltman, E.B. \& Ghigo, F.D., 1998, AJ, 93, 1220

Nakanishi, K., Saito, M. Furuya, R.S., Shinnaga, H. \& Momose, M., 2003, IAUC, 8060

Palla, F. \& Stahler, S.W., 1999, ApJ, 525, 772

Persson, S. E., D.C. Murphy, W. Krzeminski, M. Roth, and Plambeck, R. L., Wright, M. C. H., Mundy, L. G., \& Looney, L. W., 1995, ApJ, 455, L189

Probst, R.G. et al. , 2003, SPIE, 4841, 411

M.J. Rieke, 1998, AJ, 116, 2475

Reid, M. J., Readhead, A. C. S., Vermeulen, R. C., \& Treuhaft, R. N., 1999, ApJ, 524, 816

Rieke, G.H., \& Lebofsky, M.J. 1985, ApJ, 288, 618

Slee, O.B. et al. , 1987, MNRAS, 227, 467

Strom, K. M., Strom, S. E., Edwards, S., Cabrit, S., \& Skrutskie, M. F. 1989, AJ, 97, 1451

Wallace, L. \& Hinkle, K., 1997, ApJS, 111, 445

Wallace, L., Hinkle, K. \& Livingston, W.C., 2001, NSO Tech. Rep. \#01-001; Tucson: National Solar Observatory

Welch, W. J. et al. , 1996, PASP, 108, 93

White, S.M., Pallavicini, R. \& Kundu, M.R., 1992, A\&A, 259, 149 


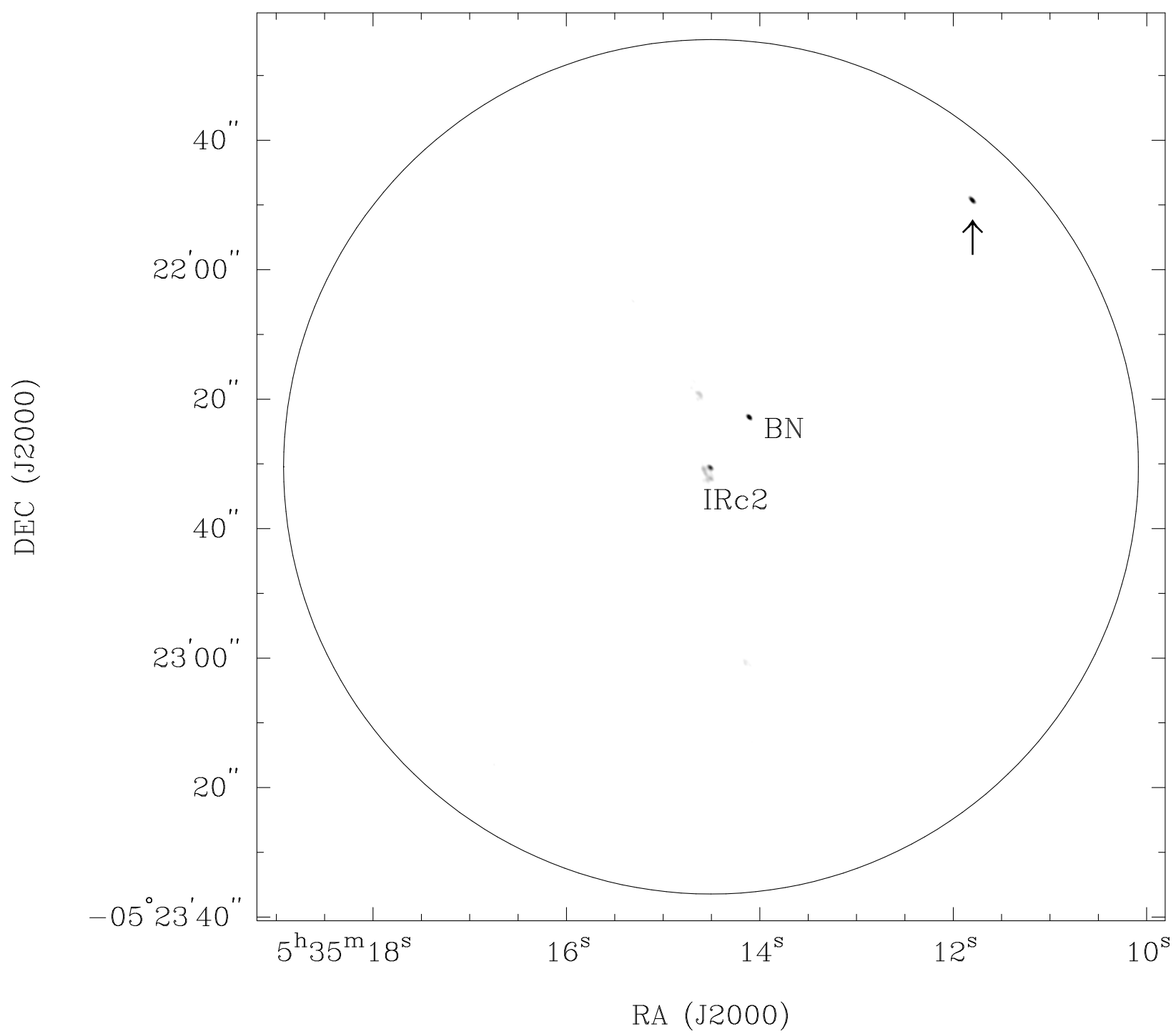

Fig. 1.- BIMA $86 \mathrm{GHz}$ continuum image of Orion-KL taken on 2003 January 20, showing the flare source at upper right. The synthesized beam is $00^{\prime \prime} 93 \times 00^{\prime \prime} 56$ at P.A. $43^{\circ}$. The halftone scale ranges from 12 to $60 \mathrm{mJy}^{\mathrm{beam}}{ }^{-1}$. The map has not been corrected for attenuation by the 2.2 FWHM primary beam, shown by the circle. Source I, the radio source associated with IRc2, is at the center the map. The faint arc just below source I is dust emission from the Orion hot core. The Becklin-Neugebauer object is $10^{\prime \prime}$ NW of source I. 


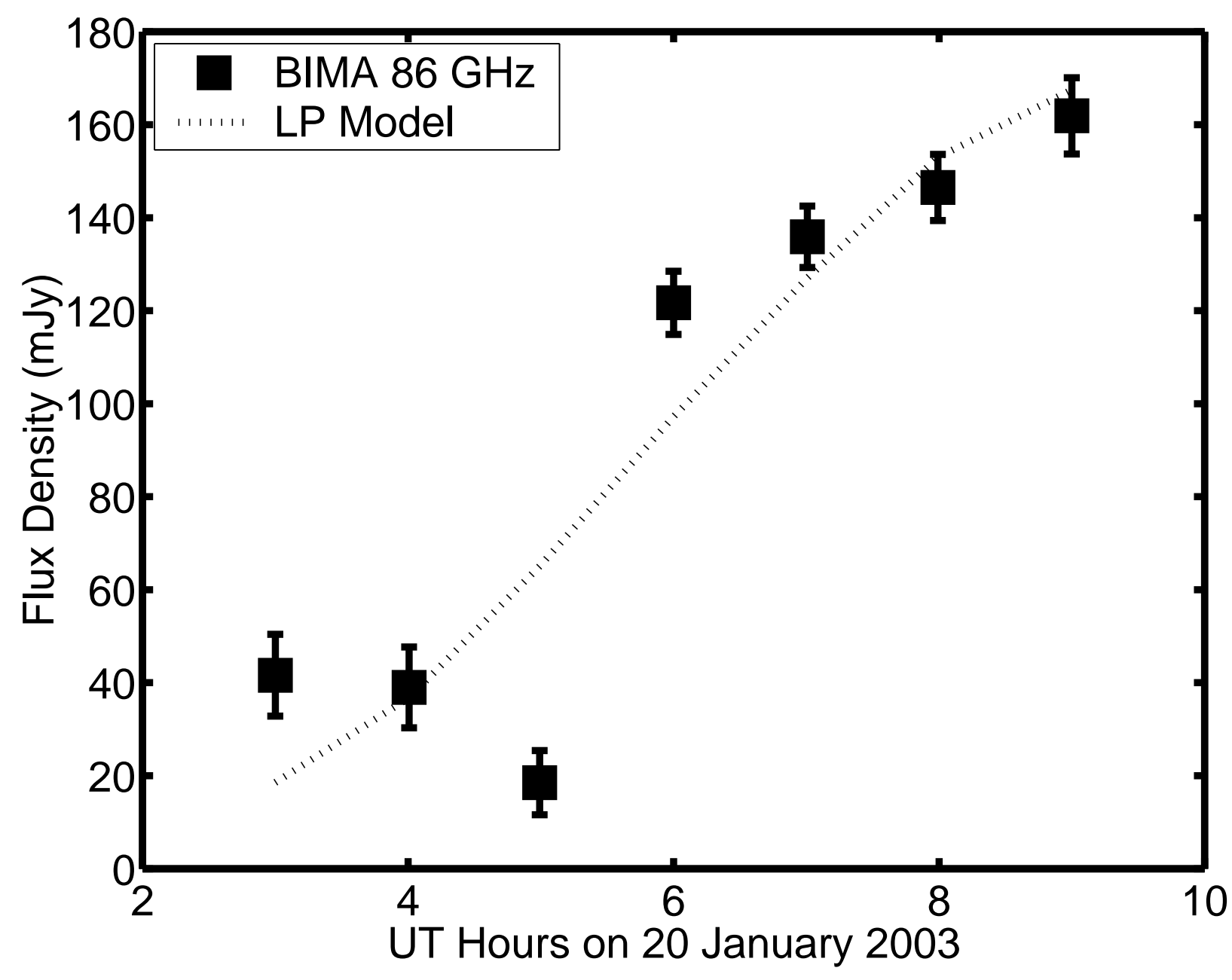

Fig. 2.- BIMA flux densities at $86 \mathrm{GHz}$ with a model of linearly polarized flux assuming $p=50 \%$ and a position angle of 107 degrees. While the polarization model follows the trend of the data, it is not an adequate fit for any position angle and polarization fraction. 


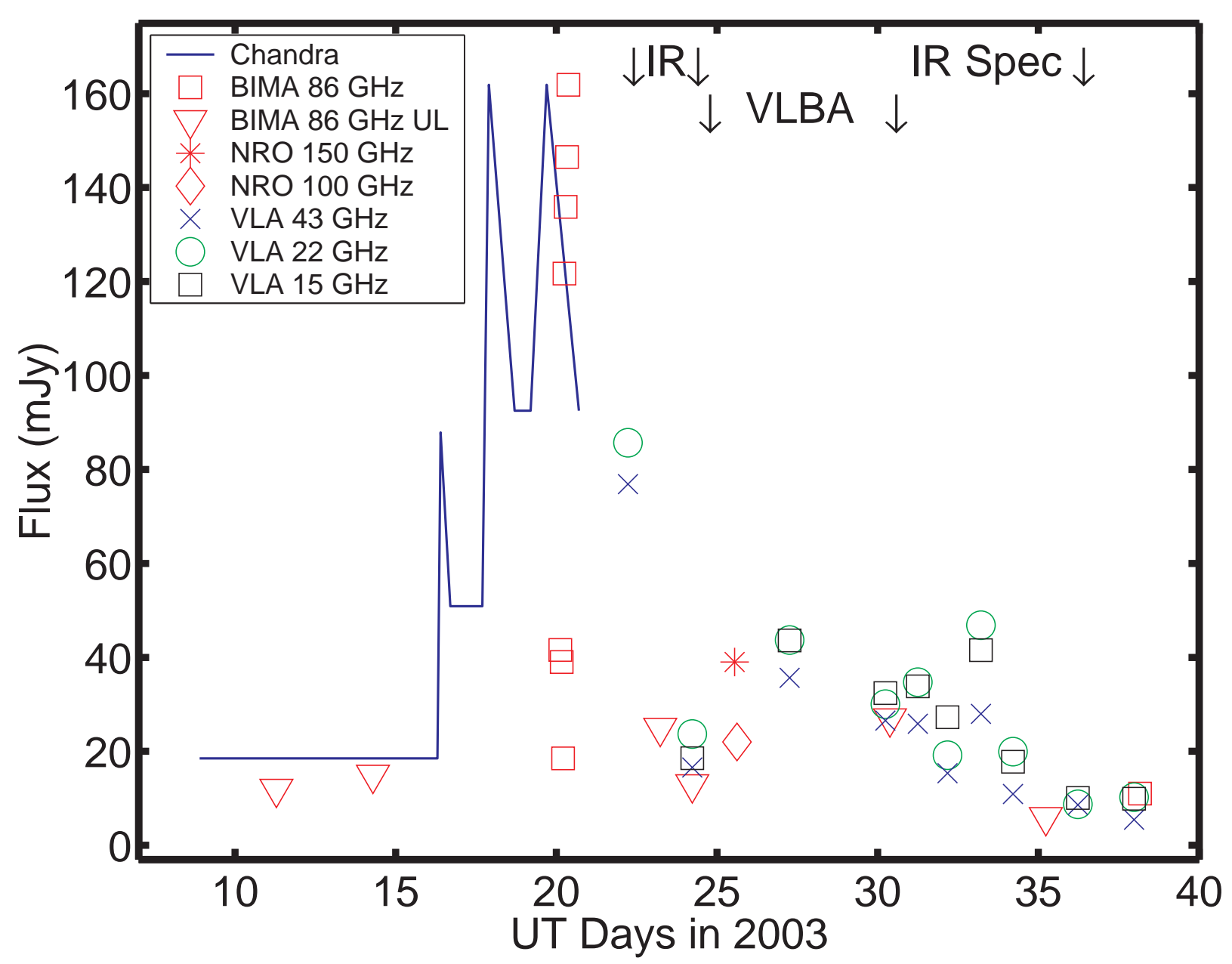

Fig. 3.- Light curve at millimeter, radio and X-ray wavelengths showing early evolution of the flare along with dates of infrared photometry ("IR" arrows), VLBA observations ("VLBA" arrows) and infrared spectroscopy ("IR Spec" arrow). Symbols are as given in the legend. The downward-facing red triangles ("BIMA $86 \mathrm{GHz}$ UL") indicate $3-\sigma$ upper limits. The X-ray light curve is from the description in Getman et al. (2003) and is given in arbitrary flux units. 


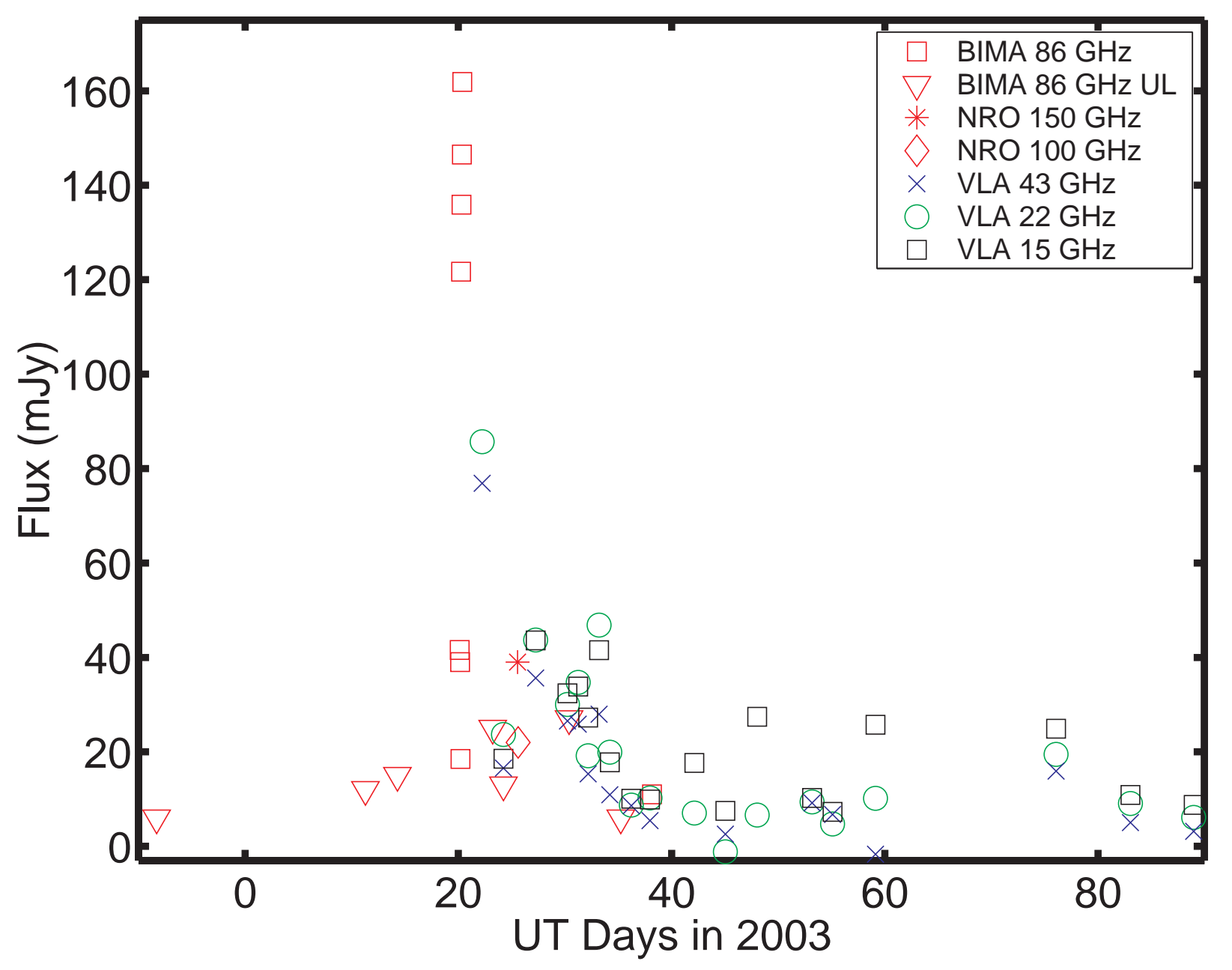

Fig. 4.- Full light curve at millimeter and radio wavelengths showing the long term evolution. Symbols are as given in the legend. The downward-facing red triangles ("BIMA 86 GHz UL") indicate $3-\sigma$ upper limits. 


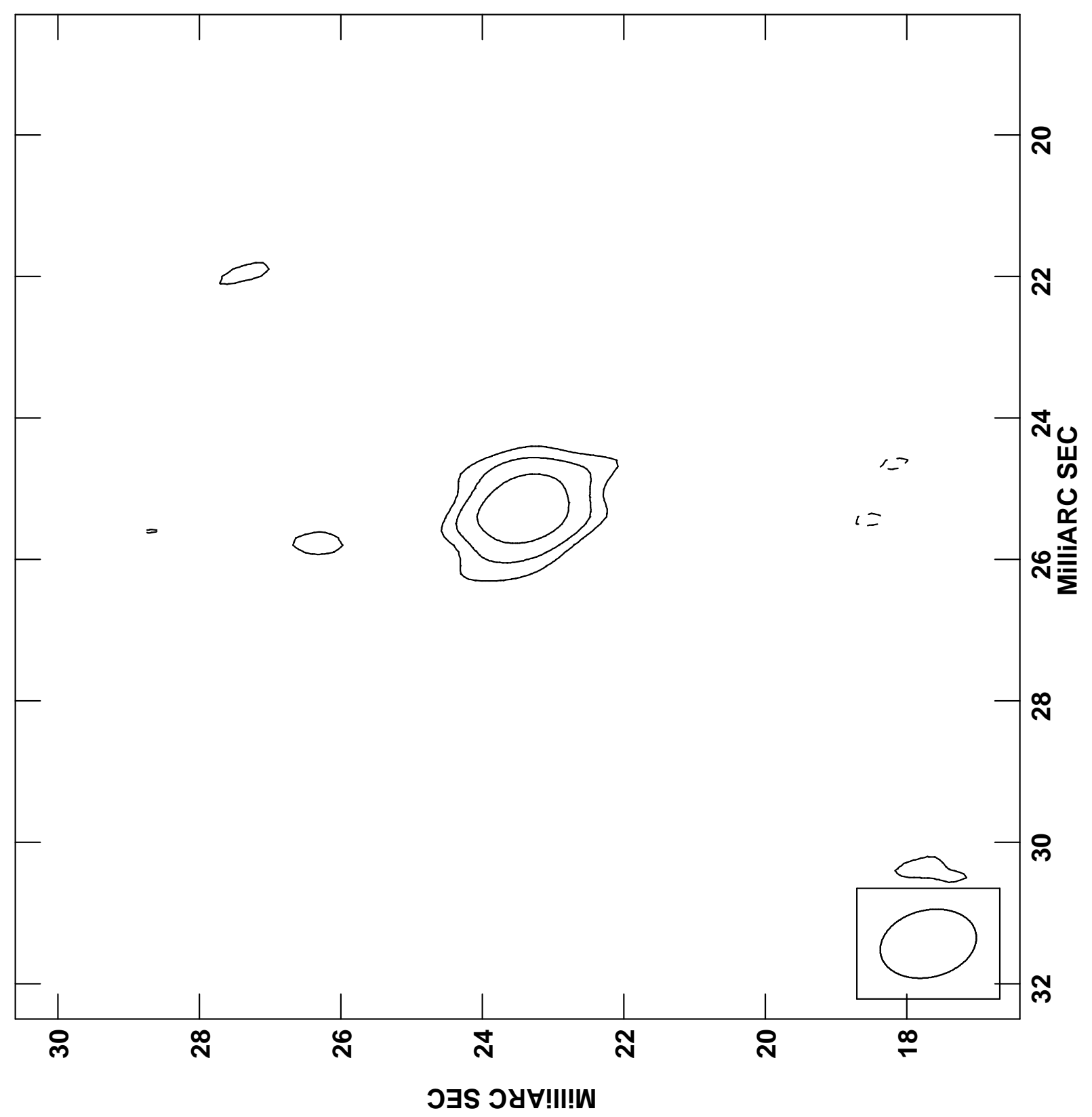

Fig. 5.- VLBA images of GMR-A at $22 \mathrm{GHz}$ obtained on 2003 January 29. Contours are -3, $3,6,12$, and 24 times the rms noise levels of $0.70 \mathrm{mJy}^{\text {beam }}{ }^{-1}$ at $22 \mathrm{GHz}$. The synthesized beam is shown in the lower left hand corner of the image. 


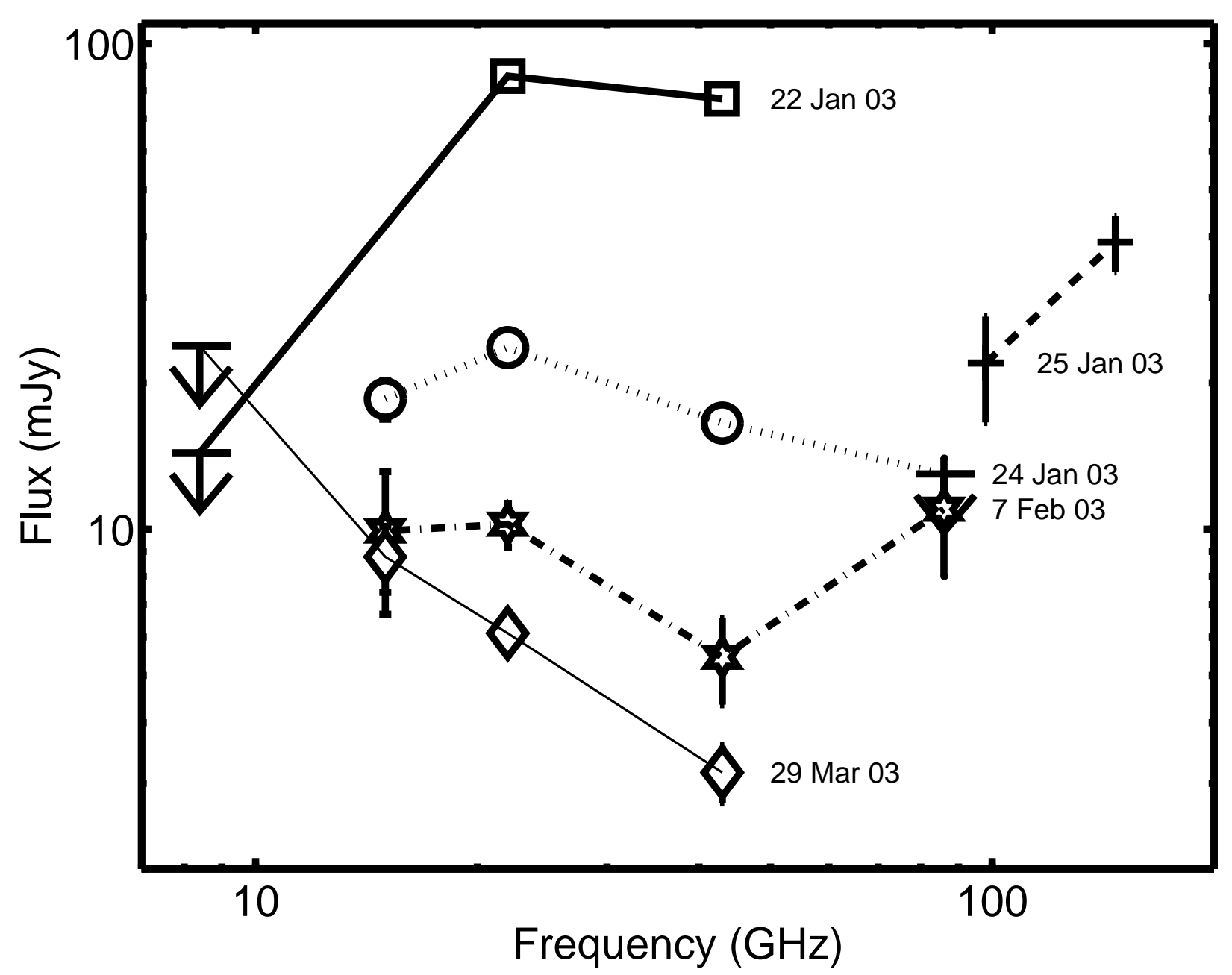

Fig. 6. - Spectra of GMR-A obtained on 2003 January 22 (squares, heavy solid line), January 24 (circles, dotted line), January 25 (crosses, dashed line), February 17 (stars, dot-dashed line) and March 29 (diamonds, light solid line). Upper limits are $3-\sigma .1-\sigma$ errors are plotted where they exceed the symbol size. The 97 and $148 \mathrm{GHz}$ data of 25 January 2003 are from Nakanishi et al. (2003). 


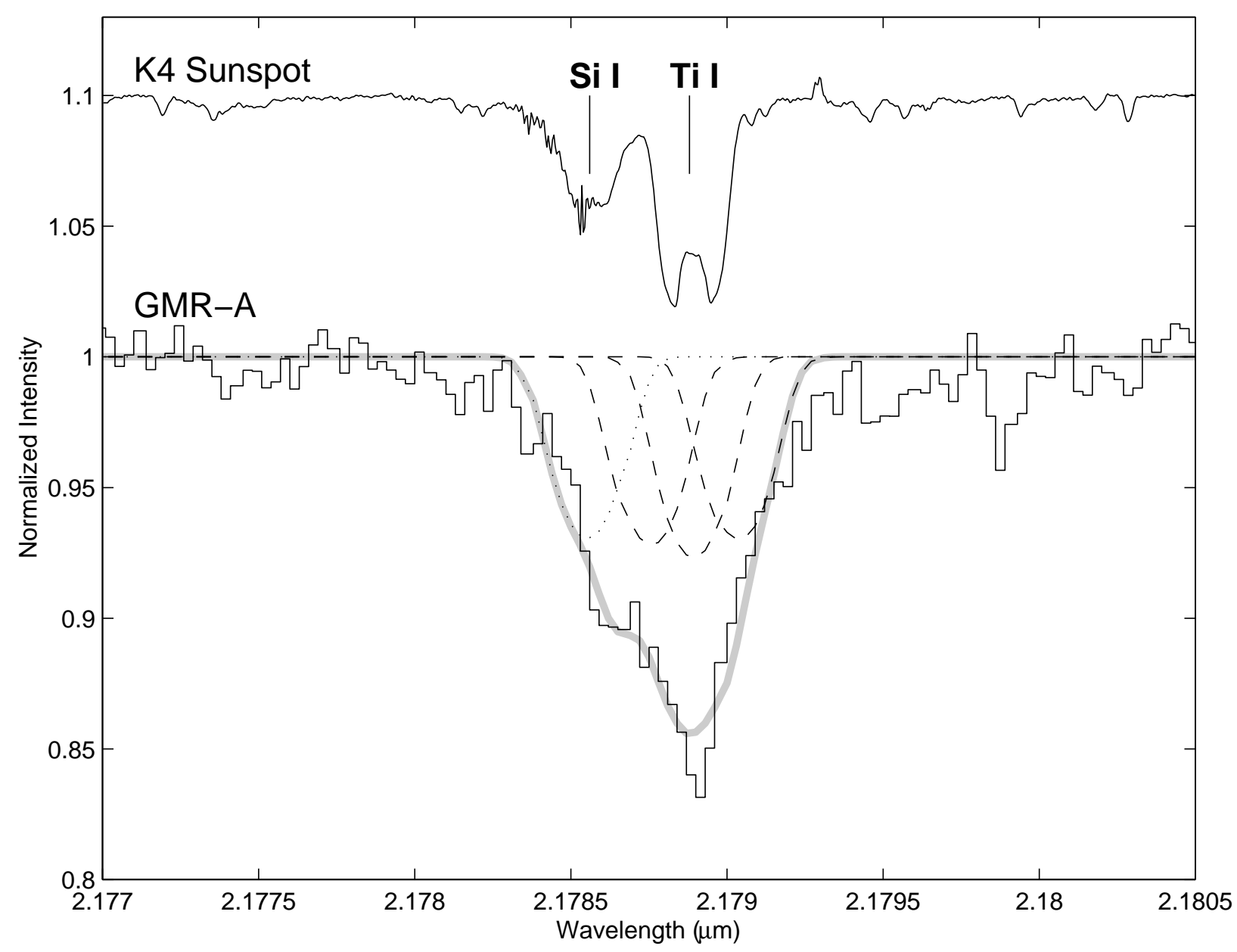

Fig. 7.- Three component Zeeman fit of the $2.17886 \mu \mathrm{m}$ transition of Ti I in GMR-A. The three Zeeman components, broadened by the instrumental profile and convolved with the rotational profile for $v \sin i=23 \mathrm{~km} \mathrm{~s}^{-1}$, are shown by dashed lines. A nearby Si I transition has been modeled in the same manner and included in the fit (dotted line). The sum of all the components is shown by the thick gray line, superimposed on the data. A high resolution spectrum of a sunspot with effective temperature similar to a K4 star and a strongly split Ti I line is shown for comparison. The splitting measured by the fit is $\Delta \lambda=0.14 \pm 0.07 \mathrm{~nm}$, corresponding to a magnetic field $3.8 \pm 2.0 \mathrm{kG}$. 
Table 1. Position of GMR-A

\begin{tabular}{llll}
\hline \hline Observation & Observing Band & \multicolumn{1}{c}{$\begin{array}{c}\text { RA } \\
(\mathrm{J} 2000)\end{array}$} & \multicolumn{1}{c}{$\begin{array}{c}\text { Dec. } \\
(\mathrm{J} 2000)\end{array}$} \\
\hline VLBA & $15 \mathrm{GHz}$ & $053511.802691 \pm 0.000001$ & $-052149.24660 \pm 0.00004$ \\
VLBA & $22 \mathrm{GHz}$ & $053511.802695 \pm 0.000001$ & $-052149.24660 \pm 0.00003$ \\
VLA & $15,22,43 \mathrm{GHz}$ & $053511.801 \pm 0.001$ & $-052149.27 \pm 0.02$ \\
BIMA & $86 \mathrm{GHz}$ & $053511.80 \pm 0.006$ & $-052149.2 \pm 0.1$ \\
IR & $2.2 \mu \mathrm{m}$ & 053511.81 & -052149.3 \\
X-Ray & $1-10 \mathrm{keV}$ & 053511.8 & -052149 \\
\hline
\end{tabular}

Note. - Units of right ascension are hours, minutes, and seconds, and units of declination are degrees, arcminutes, and arcseconds. 
Table 2. BIMA Fluxes for GMR-A

\begin{tabular}{cr}
\hline \hline Day & $\begin{array}{c}S_{86} \\
(\mathrm{mJy})\end{array}$ \\
\hline-8.29 & $<6$ \\
11.29 & $<12$ \\
14.29 & $<15$ \\
20.12 & $42 \pm 9$ \\
20.17 & $39 \pm 9$ \\
20.21 & $18 \pm 7$ \\
20.25 & $122 \pm 7$ \\
20.29 & $136 \pm 7$ \\
20.33 & $146 \pm 7$ \\
20.38 & $162 \pm 8$ \\
23.23 & $<25$ \\
24.23 & $<13$ \\
30.38 & $<27$ \\
35.23 & $<6$ \\
38.16 & $11 \pm 3$ \\
\hline
\end{tabular}

Note. - Upper limits are $3 \sigma$ corrected for attenuation by the primary beam. 
Table 3. VLA Fluxes for GMR-A

\begin{tabular}{|c|c|c|c|c|c|c|c|}
\hline UT Day & $\begin{array}{c}S_{8.4} \\
(\mathrm{mJy})\end{array}$ & $\begin{array}{c}S_{15} \\
(\mathrm{mJy})\end{array}$ & $\begin{array}{c}S_{22} \\
(\mathrm{mJy})\end{array}$ & $\begin{array}{c}S_{43} \\
(\mathrm{mJy})\end{array}$ & $\begin{array}{c}V_{15} \\
(\mathrm{mJy})\end{array}$ & $\begin{array}{c}V_{22} \\
(\mathrm{mJy})\end{array}$ & $\begin{array}{c}V_{43} \\
(\mathrm{mJy})\end{array}$ \\
\hline 22.23 & $<33$ & & $85.7 \pm 0.7$ & $76.9 \pm 1.0$ & & $<1.4$ & $<2.8$ \\
\hline 24.23 & & $18.5 \pm 1.7$ & $23.7 \pm 0.6$ & $16.6 \pm 0.5$ & $<2.6$ & $-1.2 \pm 0.3$ & $-1.9 \pm 0.5$ \\
\hline 27.26 & $\ldots$ & $43.6 \pm 1.6$ & $43.7 \pm 0.5$ & $35.7 \pm 0.4$ & $<1.4$ & $-2.2 \pm 0.2$ & $-3.4 \pm 0.5$ \\
\hline 30.24 & $\ldots$ & $32.4 \pm 1.7$ & $30.1 \pm 0.8$ & $26.6 \pm 0.5$ & $<2.0$ & $<1.0$ & $<2.3$ \\
\hline 31.25 & $\ldots$ & $33.8 \pm 1.6$ & $34.7 \pm 0.6$ & $25.9 \pm 0.5$ & $<1.9$ & $-0.6 \pm 0.2$ & $<1.9$ \\
\hline 32.17 & 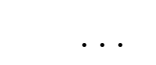 & $27.3 \pm 1.6$ & $19.2 \pm 0.6$ & $15.3 \pm 0.4$ & $<1.8$ & $-1.1 \pm 0.2$ & $<2.1$ \\
\hline 33.21 & 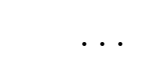 & $41.6 \pm 1.6$ & $46.8 \pm 0.6$ & $28.0 \pm 0.5$ & $-1.3 \pm 0.3$ & $-1.8 \pm 0.2$ & $-1.9 \pm 0.4$ \\
\hline 34.21 & $<31$ & $17.8 \pm 1.0$ & $19.9 \pm 0.3$ & $10.9 \pm 0.3$ & $-1.2 \pm 0.2$ & $-1.0 \pm 0.1$ & $-0.9 \pm 0.3$ \\
\hline 36.23 & $\ldots$ & $10.1 \pm 2.5$ & $8.7 \pm 1.3$ & $8.6 \pm 0.5$ & $<1.8$ & $<3.1$ & $<1.9$ \\
\hline 37.98 & $\ldots$ & $9.9 \pm 3.2$ & $10.2 \pm 1.1$ & $5.5 \pm 1.1$ & $<4.0$ & $<1.2$ & $<1.6$ \\
\hline 42.13 & $\ldots$ & $17.7 \pm 2.1$ & $7.1 \pm 0.6$ & $<1.0$ & $<1.4$ & $-0.6 \pm 0.1$ & $<1.3$ \\
\hline 45.04 & $<36$ & $7.5 \pm 2.1$ & $<2.9$ & $2.6 \pm 0.5$ & $<1.8$ & $<1.8$ & $<1.6$ \\
\hline 48.02 & $<33$ & $27.4 \pm 1.9$ & $6.6 \pm 0.7$ & $<1.7$ & $<3.5$ & $<1.5$ & $<1.3$ \\
\hline 53.17 & $<21$ & $10.2 \pm 1.6$ & $9.4 \pm 0.6$ & $9.2 \pm 0.3$ & $-1.2 \pm 0.2$ & $<0.9$ & $-1.0 \pm 0.2$ \\
\hline 55.10 & & $7.3 \pm 1.8$ & $4.7 \pm 0.7$ & $6.7 \pm 0.3$ & $<1.5$ & $<0.9$ & $<1.2$ \\
\hline 59.12 & $<26$ & $25.8 \pm 1.9$ & $10.2 \pm 0.8$ & $<1.5$ & $-0.7 \pm 0.2$ & $-1.0 \pm 0.1$ & $<1.1$ \\
\hline 76.06 & $<18$ & $24.9 \pm 1.1$ & $19.5 \pm 0.5$ & $15.9 \pm 0.3$ & $<1.2$ & $-0.5 \pm 0.1$ & $-1.9 \pm 0.3$ \\
\hline 83.04 & $<21$ & $10.9 \pm 1.0$ & $9.1 \pm 0.4$ & $5.0 \pm 0.2$ & $-0.9 \pm 0.1$ & $-0.9 \pm 0.1$ & $<1.2$ \\
\hline 88.96 & $<36$ & $8.8 \pm 1.4$ & $6.1 \pm 0.5$ & $3.2 \pm 0.4$ & $<1.0$ & $-0.9 \pm 0.1$ & $-0.6 \pm 0.1$ \\
\hline
\end{tabular}

Note. $-S_{f}$ and $V_{f}$ are the total intensity and circularly polarized flux density at frequency $f$. Upper limits given for $V_{f}$ are $2 \sigma$ limits on the absolute value. UT days are given for the year 2003 . 
Table 4. Infrared Photometry

\begin{tabular}{|c|c|c|c|c|c|c|}
\hline Star & Date & Telescope & $\mathrm{J}$ & $\mathrm{H}$ & $\mathrm{K}_{S}$ & Reference \\
\hline GMR-A & 02/09/99 & Keck/NIRC & & 11.9 & 9.6 & HC2000 \#573 \\
\hline GMR-A & $01 / 22 / 03$ & Keck/NIRC & 16.0 & 11.98 & 9.61 & This paper \\
\hline GMR-A & $01 / 24 / 03$ & CTIO & 15.8 & 11.9 & 9.7 & This paper \\
\hline Star W & $02 / 09 / 99$ & Keck/NIRC & $\ldots$ & 10.5 & 9.9 & HC2000 \#554 \\
\hline Star W & $01 / 22 / 03$ & Keck/NIRC & 11.1 & 10.38 & 9.75 & This paper \\
\hline Star W & $01 / 24 / 03$ & CTIO & 11.2 & 10.3 & 9.8 & This paper \\
\hline Star S & $02 / 09 / 99$ & Keck/NIRC & $\ldots$ & 12.0 & 11.6 & HC2000 \#555 \\
\hline Star S & $01 / 22 / 03$ & Keck/NIRC & 12.7 & 12.1 & 11.5 & This paper \\
\hline Star S & $01 / 24 / 03$ & CTIO & 12.7 & 12.0 & 11.6 & This paper \\
\hline
\end{tabular}

Note. - Star S is the bright K band object $6^{\prime \prime}$ South of GMR-A. Star W is a bright K band object 6 " South and 20" West of GMR-A. HC2000 refers to Hillenbrand \& Carpenter (2000). 